\title{
Image Classification on NXP i.MX RT1060 using Ultra-thin MobileNet DNN
}

\author{
Saurabh Ravindra Desai \\ Department of ECE \\ saudesai@iu.edu
}

Debjyoti Sinha

Department of ECE

Purdue School of Engineering and Tech. Purdue School of Engineering
Indianapolis, USA

\author{
debsinha@iu.edu
}

\author{
Mohamed El-Sharkawy \\ Department of ECE
}

$\sqrt{2}$ 


\section{PRIOR WORK}

There are some deep neural networks which use depthwise separable convolutions and separable convolutions instead of the normal standard convolutions. Some of the deep neural networks using these convolutions are MobileNet V1 [3], MobileNet V2 [5], Enhanced Hybrid MobileNet [14] CondenseNet [11], ShuffleNet [8], and Xception [9]. The MobileNet V1 architecture utilizes the depthwise separable convolutions to decrease the average computation overhead to about one-eighth of the computation overhead incurred when standard convolutions are used. In a depthwise layer, each input channel is filtered separately and they are followed by a $1 \subset 1$ pointwise convolution layer where all the outputs are linearly integrated. So, the process of filtering and the process of combining takes place in two different steps. MobileNet V2 [5] is an improved DNN architecture as compared to the MobileNet V1 [3] based on the model size. It utilizes bottleneck depthwise separable convolutions with residuals. It has got a 32 filter fully convolution layer, succeeded by 19 residual layers. The utilization of these residual layers prevents data from being mutilated and also makes the model more compact. The Enhanced Hybrid MobileNet [14] is also an improvement over the existing baseline model. In this model, the depth multiplier [14] hyperparameter was introduced and the average pooling layers were substituted by Max pooling and Fractional max pooling layers [14] with different strides. Different enhanced hybrid models were generated as the stride values were changed. Some achieved better accuracy and some achieved lesser model size as compared to the MobileNet V1. CondenseNet [11] uses some special convolutions called the group convolutions $[8,11]$ mainly to reduce the computation cost by dividing the input features into mutually exclusive $G$ groups, and they produce their outputs, ultimately decreasing the overall computational cost by a factor of G. Another model, the ShuffleNet [8] also uses these group convolutions. Each unit is composed of $1 \times 1$ pointwise convolution layers, channel shuffle, $3 \times 3$ depthwise convolutions, and $1 \times 1$ group convolutions. The channels in a group are divided into subgroups and then they are fed into the next layer with different subgroups, which makes the input channel and output channel perfectly related to each other. This model has very less number of MFLOPS compared to many other renowned DNN models. The Xception [9] model makes use of depthwise separable convolutions with residual connections. It was an improvement over the Inception V3 [4, 9] architecture, showing that it could achieve more gains in classification performance with the same number of parameters as the Inception module.

\section{THE DEEP NEURAL NETWORK ULTRA-THIN MOBILENET}

The Ultra-thin MobileNet is an enhanced MobileNet [3] architecture which has been developed by introducing some modifications on the existing baseline MobileNet V1 architecture. It is faster, more accurate and less memory intensive as compared to the baseline MobileNet V1 model. We propose some improvements to the existing baseline MobileNet V1
TABLE I

NETWORK ARCHITECTURE AFTER INTRODUCING IMPROVEMENT 1

\begin{tabular}{|l|l|l|}
\hline Layer/Strides & Output Shape & No. of Parameter \\
\hline Input layer & $32,32,3$ & 0 \\
\hline Conv/s2 & $16,16,32$ & 864 \\
\hline Separable Conv/s1 & $16,16,32$ & 1312 \\
\hline Separable Conv/s2 & $8,8,64$ & 2336 \\
\hline Separable Conv/s1 & $8,8,128$ & 8768 \\
\hline Separable Conv/s2 & $4,4,128$ & 17536 \\
\hline Separable Conv/s1 & $4,4,256$ & 33920 \\
\hline Separable Conv/s2 & $2,2,256$ & 67840 \\
\hline Separable Conv/s1 & $2,2,512$ & 133376 \\
\hline Separable Conv/s1 & $2,2,512$ & 133376 \\
\hline Separable Conv/s1 & $2,2,512$ & 266752 \\
\hline Separable Conv/s1 & $2,2,512$ & 266752 \\
\hline Separable Conv/s1 & $2,2,512$ & 266752 \\
\hline Separable Conv/s2 & $1,1,512$ & 266752 \\
\hline Separable Conv/s1 & $1,1,1024$ & 528896 \\
\hline Global avg. pool/s1 & $1,1,1024$ & 0 \\
\hline FC and Softmax/s1 & $1,1,10$ & 10250 \\
\hline
\end{tabular}

architecture to obtain the Ultra-thin MobileNet model that is better in terms of size, computation time, accuracy and is easily deployable in resource-constrained microprocessor units. These are the following:

\section{A. Improvement 1- Replacing Depthwise Separable convolu- tions [3, 5, 8, 14] by Separable convolutions [9]}

Depthwise separable convolution [3, 5, 8, 14] contains depthwise convolution layers where each input channel is filtered individually, succeeded by pointwise convolutions where the outputs are integrated linearly. The depthwise convolution and the pointwise convolution layers are defined independently in the MobileNet v1 [3] model, but in our model, the depthwise layer and the pointwise layer are combined into one layer and defined as Separable convolutions [9]. In the python program, the depthwise initializer, depthwise regularizer, depthwise constraint, and pointwise initializer, pointwise regularizer, and pointwise constraint are defined inside the same init() function. There is no separate definition for the pointwise convolutions. The role of the separable convolutions is similar to the depthwise separable convolutions, the advantage being a reduction in the number of layers from 28 to 14 , decreased model size, reduced total number of parameters and very less average computation-time-per-epoch as compared to the MobileNet V1 architecture. The model size is now $26.1 \mathrm{MB}$ which is $13 \mathrm{MB}$ less as compared to the baseline MobileNet V1 [3] size and the total number of parameters is $2.1 \mathrm{M}$ which is $1.2 \mathrm{M}$ less than the baseline MobileNet V1 [3]. The average computation time is $21 \mathrm{~s}$. TABLE I. shows the architecture after introducing this modification.

\section{B. Improvement 2- Utilizing Random Erasing data augmen- tation technique $[13,17]$}

Data augmentation techniques [17] are used to augment the size of the training dataset by creating alternative versions of the images inside that dataset. In Random erasing [13] technique, we randomly select rectangular regions in an image I in a mini-batch, delete the pixels of that region and substitute 
TABLE II

NETWORK ARCHITECTURE AFTER INTRODUCING IMPROVEMENTS 1,2,3 AND 4

\begin{tabular}{|l|l|l|}
\hline Layer/Strides & Output Shape & No. of Parameter \\
\hline Input layer & $32,32,3$ & 0 \\
\hline Conv/s2 & $16,16,32$ & 864 \\
\hline Separable Conv/s1 & $16,16,32$ & 1312 \\
\hline Separable Conv/s2 & $8,8,64$ & 2336 \\
\hline Separable Conv/s1 & $8,8,128$ & 8768 \\
\hline Separable Conv/s2 & $4,4,128$ & 17536 \\
\hline Separable Conv/s1 & $4,4,256$ & 33920 \\
\hline Separable Conv/s2 & $2,2,256$ & 67840 \\
\hline Separable Conv/s2 & $1,1,512$ & 133376 \\
\hline Separable Conv/s1 & $1,1,728$ & 377344 \\
\hline Global avg. pool/s1 & $1,1,728$ & 0 \\
\hline FC and Softmax/s1 & $1,1,10$ & 7290 \\
\hline
\end{tabular}

it with random values. When some portion of an item in an image is occluded, a model can fail to recognize the item from its global structure due to the absence of good generalization ability. This technique enhances the generalization ability of a model. In our model, the parameter values used are:

1. Probability of erasing $\mathrm{p}=0.5$.

2. Max. erasing area ratio $S_{h}=0.02$.

3. Min. erasing area ratio $S_{1}=0.4$.

4. Range of Erasing aspect ratio $=[0.3,3.33]$.

5. Erasing aspect area ratio $r_{\mathrm{e}}=0.3$.

Area of the original image $=\mathrm{S}$.

Erased area $=\mathrm{S}_{\mathrm{e}}$

Erasing area ratio $=\underline{\mathrm{Se}}_{\mathrm{S}}$

As a result of this, the accuracy increases slightly and the overfitting decreases to some extent.

\section{Improvement 3- Layers with similar output shape [3] are eliminated}

We make our network shallow by removing the layers with redundant output shapes. The layers 9 to 13 in TABLE I. which have the output shape of $(2,2,512)$ are eliminated. These layers contribute to $41 \%$ of the total number of parameters. The model size is now 9.9 MB which is almost $30 \mathrm{MB}$ less than the size of baseline MobileNet v1.

\section{Improvement 4- Changing the channel depth}

We change the depth of the channel in the last separable convolution block from 1024 to 728 [9]. The block with the channel depth 1024 results in about $0.5 \mathrm{M}$ parameters. When we modify the channel depth to 728 , the number of parameters reduces by $0.2 \mathrm{M}$, which further reduces the model size to 8 MB.

TABLE II. shows the resulting network after introducing the improvements 1,2,3 and 4 .

E. Improvement 5- Substituting Swish activation function [16, 19, 20] instead of ReLU [16, 19]

ReLU is a standard non-linear activation function that performs better than most of the other activation functions for almost all the DNN models trained on any dataset. The baseline MobileNet v1 uses ReLU [16, 19] as the activation

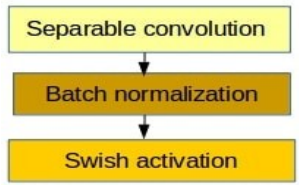

Fig. 1. Block with Separable convolution followed by Batch normalization and Swish activation

TABLE III

THE EFFECT OF USING DIFFERENT WIDTH MULTIPLIER VALUES ON THE MODEL

\begin{tabular}{|l|l|l|l|}
\hline Width multiplier $\alpha$ & Model size & Accuracy & Parameters \\
\hline 0.75 & $4.8 \mathrm{MB}$ & $85.15 \%$ & $3,76,270$ \\
\hline 0.69 & $3.9 \mathrm{MB}$ & $84.32 \%$ & $3,19,095$ \\
\hline 0.60 & $3.0 \mathrm{MB}$ & $83.99 \%$ & $2,43,167$ \\
\hline 0.50 & $2.2 \mathrm{MB}$ & $81.86 \%$ & $1,72,130$ \\
\hline
\end{tabular}

function. If we replace it by the Swish activation function $[15,18,19]$, we get a better accuracy which is $85.60 \%$. It is a smooth, non-monotonic, lower bounded and upper unbounded activation function. It is mathematically defined as:

$$
f(x)=x \cdot \sigma(\beta . x)=\frac{x}{1+e^{-x}}
$$

$\sigma=$ Sigmoid function and $\beta=$ Trainable parameter. When, the value of $\beta=0$, Swish becomes a linear function that is $f(x)=$ $\frac{x}{2}$. When $\beta \rightarrow$ Swish starts behaving like the standard ReLU activation function. Our model has the best accuracy when we set $\beta=1$. In this case, Swish becomes the Sigmoid-weighted Linear Unit(SiL) [20, 23]. Fig. 1. shows the use of the Swish activation in our model.

\section{F. Improvement 6- Setting the width multiplier [3, 5, 15] hyperparameter value}

The width multiplier $\alpha[3,5,14]$ is a hyperparameter that is used to make a model slim uniformly at each layer. It reduces the number of input and output channels and makes the model thinner and faster. The width multiplier $\alpha$ should have values in the range 0 to 1 . The total number of parameters decreases quadratically [3] as we reduce the value of $\alpha$ from 1 to 0 . TABLE III. shows the model size, accuracy and the number of parameters we get with different values of $\alpha$. It is evident from the table that as we decrease the value of the width multiplier, the accuracy starts to fall and it goes below the baseline MobileNet V1 accuracy which is $84.30 \%$.

We observe, that as we reduce the value of $\alpha$, the accuracy decreases and falls below the baseline level accuracy [3] of $84.30 \%$. After putting different values of $\alpha$ in our python code, we discover that when $\alpha=0.69$, we get an accuracy of $84.32 \%$ which is a little above the baseline accuracy. We are keeping the value of $\alpha=0.69$ because we want to develop a network that has less model size and computation time than the baseline MobileNet with almost the same level of accuracy. In the process, the overfitting problem reduces to a large extent We call this DNN model as the Ultra-thin MobileNet. TABLE IV. shows the Ultra-thin MobileNet architecture with all the 
TABLE IV

UltRA-THIN MOBILENET

\begin{tabular}{|l|l|l|}
\hline Layer/Strides & Output Shape & No. of Parameter \\
\hline Input layer & $32,32,3$ & 0 \\
\hline Conv/s2 & $16,16,22$ & 594 \\
\hline Separable Conv/s1 & $16,16,22$ & 682 \\
\hline Separable Conv/s2 & $8,8,44$ & 1166 \\
\hline Separable Conv/s1 & $8,8,88$ & 4268 \\
\hline Separable Conv/s2 & $4,4,88$ & 8536 \\
\hline Separable Conv/s1 & $4,4,176$ & 16280 \\
\hline Separable Conv/s2 & $2,2,176$ & 32560 \\
\hline Separable Conv/s2 & $1,1,353$ & 63712 \\
\hline Separable Conv/s1 & $1,1,502$ & 180383 \\
\hline Global avg. pool/s1 & $1,1,502$ & 0 \\
\hline FC and Softmax/s1 & $1,1,10$ & 5030 \\
\hline
\end{tabular}

layers, the output shape, and parameters associated with each layer.

\section{G. Improvement 7- Choosing a good optimizer}

We obtain the best accuracy by using Nadam [20] as the optimizer. Nadam [20] combines the effect of RMSProp [21], Adam [12, 24] and Nesterov momentum [10, 23]. It is an efficient optimizer which interrupts the search in the direction of oscillations and speeds up the search towards the direction of the minima. It is better than Momentum [27] and SGD [25] optimizers as it does not drastically overshoot around the minima.

\section{HARDWARE AND SOFTWARE REQUIREMENTS}

(a) NVIDIA Geforce RTX 1080Ti GPU

(b) Intel i9 9th generation processor (32GB RAM)

(c) NXP i.MX RT1060 MCU

(d) Anaconda Navigator 2.0

(d) Python IDE- Spyder v3.6

(e) Keras v2.2.0

(f) Tensorflow-gpu v1.11.0

(g) Livelossplot package from PyPI

(h) MCU Xpresso SDK

(i) Microsoft Visual Studio Code IDE

(j) Tera Term

\section{IMPLEMENTATION}

\section{A. Developing the DNN model}

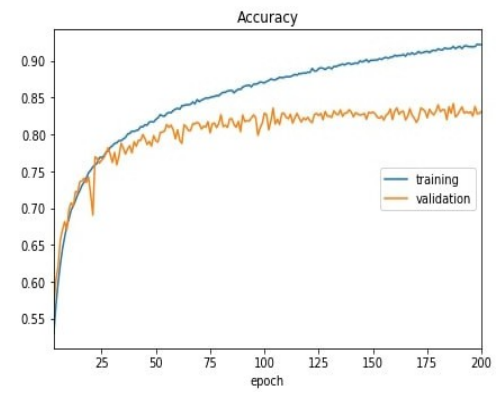

Fig. 2. MobileNet v1 baseline accuracy plot

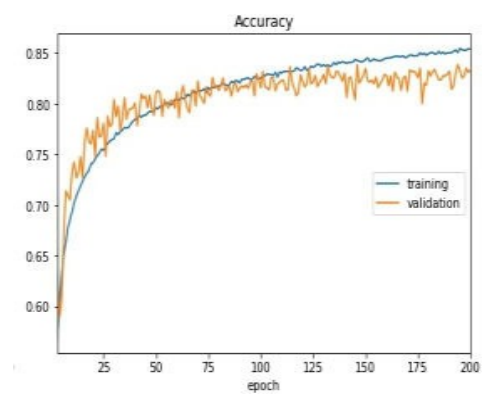

Fig. 3. Ultra-thin MobileNet accuracy plot

TABLE V

ULtRA-THIN MOBILENET v1 FeATURES

\begin{tabular}{|l|l|}
\hline Accuracy & $84.32 \%$ \\
\hline Model size & $3.9 \mathrm{MB}$ \\
\hline Avg. Computation time per epoch & $16 \mathrm{~s}$ \\
\hline No. of parameters & $3,19,095$ \\
\hline
\end{tabular}

We have trained the baseline MobileNet V1 from scratch over the CIFAR-10 dataset in 32 batches and 1563 steps- perepoch for 200 epochs. The model size is $39.1 \mathrm{MB}$, the accuracy obtained is $84.30 \%$, the number of parameters is 3.2 $\mathrm{M}$ and the average computation time is $31 \mathrm{~s}$. Fig. 2. shows the accuracy plot for the baseline version. The parameters are saved and loaded from a checkpoint file. Then we introduce the above-mentioned improvements into the baseline version to obtain an enhanced DNN which we call as the Ultra-thin MobileNet. It is trained for 200 epochs in 32 batches and the accuracy and the loss updates after each epoch is viewed graphically using the livelossplot package. NVIDIA Geforce RTX 1080Ti GPU is the hardware used for training. It is also tested on the CIFAR-10 dataset for class prediction. Fig 3. shows the plot of accuracy for the Ultra-thin MobileNet. TABLE V. depicts the features of this efficient network.

\section{B. Converting Model to TensorFlow Lite}

The NXP eIQ is a machine learning software development environment to develop machine learning applications for embedded processors such as i.MX RT crossover processors. The eIQ software includes inference engines, neural network compilers, and optimized libraries. TensorFlow Lite is one of the inference engines supported by eIQ software with high performance and optimized memory utilization than TensorFlow. TFLiteConverter takes an existing model in the keras framework and generates the TensorFlow Lite FlatBuffer file (.tflite). The Python API for TFLiteConverter allows custom objects such as activation functions, loss functions, etc to be passed during the conversion process. The IDE used for this conversion process is Microsoft Visual Studio Code (VS Code).

\section{Deployment on NXP i.MXRT1060}

eIQ software is delivered as middleware in the latest MCUXpresso SDK for NXP i.MXRT 1060. The MCUXpresso 
SDK includes updated eIQ software platform and demos. Package contains a label image example for TensorFlow Lite, which is imported in MCUXpresso. The SDK also supports UART debug Console to debug the application on the TeraTerm serial emulator. This example runs a deep learning model on incoming frames from a camera for image classification. TensorFlow Lite FlatBuffer file (.tflite) is then converted into a $\mathrm{C}$ array header file (.h) that can be imported into an embedded project. This header file is then used to load the model in the code using API call. Application is then built to compile the code and create an executable for the i.MX platform. Fig. 4. is a snapshot of the i.MX RT1060 hardware we are using to run our model. The application is then debugged on the TeraTerm for output.

\section{RESULTS}

TABLE $\mathrm{V}$ shows that the Ultra-thin MobileNet model is better than the baseline MobileNet model in terms of size, accuracy, and computation time (average time cost per epoch). TABLE VI compares our architecture with the other architectures like the MobileNet V2, Effnet [28], ShuffleNet $2 x$ [29], Inception V1 [4], Inception V3, Xception, etc in terms of accuracy and the average computation time per epoch when they are trained using the CIFAR-10 dataset. The Ultra-thin MobileNet achieves the highest accuracy of $84.32 \%$ among these models. Also, it has an average computation time of $16 \mathrm{~s}$ which is better than all the other DNN models. TABLE VII compares our model with other DNN models like the MobileNet, MobileNet V2, Xception V1, ShuffleNet $2 \mathrm{x}$, Inception V1 and Inception V3 models in terms of the total number of parameters(millions) when they are trained on the CIFAR-10 dataset. In this case, also, our model is having a fewer number of total parameters than all other DNN models compared to in the table. Our model has about $0.3 \mathrm{M}$ parameters which is very less than the number of parameters in every other DNN model. Fig. 5. demonstrates the results of the image classification application on the NXP i.MX RT1060 board when the Ultra-thin MobileNet DNN model is run on it. The results are visible on the TeraTerm terminal emulator. The CIFAR 10-dataset mainly has 10 classes of images. The processor recognizes these classes of images and classifies them into one of the 10 categories in approximately $120 \mathrm{~ms}$ (average inference time). For each category, the processor assigns a probability value and the highest probability is displayed as the recognized category. For example, if we feed in, the image of a ship, the TeraTerm serial emulator shows the inference time in milliseconds first and then shows the detected category as a ship with a percentage value.

\section{CONCLUSION}

We have developed a new DNN architecture called the Ultra-thin MobileNet by introducing some improvements to the baseline MobileNet architecture. To make the model compact, we have used Separable convolutions instead of Depthwise Separable Convolution layers, eliminate the layers with redundant output shape, change the channel depth at
TABLE VI

COMPARISON WITH OTHER DNNS BASED ON ACCURACY AND THE COMPUTATION TIME ON THE CIFAR-10 DATASET

\begin{tabular}{|l|c|l|}
\hline Model & Accuracy & Computation time \\
\hline Ultra-thin MobileNet & $84.32 \%$ & $16 \mathrm{~s}$ \\
\hline MobileNet & $84.30 \%$ & $31 \mathrm{~s}$ \\
\hline MobileNet V2 & $73.98 \%$ & $18 \mathrm{~s}$ \\
\hline Inception V1 & $75.21 \%$ & $63 \mathrm{~s}$ \\
\hline Inception V3 & $79.76 \%$ & $61 \mathrm{~s}$ \\
\hline EffNet & $80.20 \%$ & $24 \mathrm{~s}$ \\
\hline Xception & $75.10 \%$ & $58 \mathrm{~s}$ \\
\hline
\end{tabular}

TABLE VII

COMPARISON WITH OTHER DNNS BASED ON THE TOTAL NUMBER OF PARAMETERS (MILLIONS) ON THE CIFAR-10 DATASET

\begin{tabular}{|l|l|}
\hline Model & Parameters \\
\hline Ultra-thin MobileNet & $0.3 \mathrm{M}$ \\
\hline MobileNet & $3.2 \mathrm{M}$ \\
\hline MobileNet V2 & $2.3 \mathrm{M}$ \\
\hline Xception V1 & $22.8 \mathrm{M}$ \\
\hline ShuffleNet 2x & $5.2 \mathrm{M}$ \\
\hline Inception V1 & $6.6 \mathrm{M}$ \\
\hline Inception V3 & $23.6 \mathrm{M}$ \\
\hline
\end{tabular}

the last layer and use the width multiplier hyperparameter with an optimum value of $\alpha=0.69$. The value of $\alpha=0.69$ is crucial here, as it reduces the size drastically, eradicates the overfitting problem, without allowing the overall accuracy of the model to fall below the baseline level. The interesting feature of this model is that when we make the model more compact, the accuracy of the model does not decrease due to the use of the Swish activation function, Random Erasing data augmentation method and Nadam optimizer. They play a very important role in maintaining the accuracy above the $84.30 \%$ mark which is the baseline MobileNet V1 accuracy. Generally, models with less depth are not very accurate as compared to the models with high depth. The size of the model is only 3.9 MB which makes it deployable into resource-constrained real-time microprocessors. The model also has a competitive accuracy of $84.32 \%$ which makes it safe and reliable for realtime image classification applications. The model is deployed into the NXP i.MX RT1060 MCU for the image classification application. This paper also proposes a method to convert a DNN image classification model trained with the Keras framework into a TensorFlow Lite format model, which is compatible with the NXP i.MX RT crossover processors. The average inference time to classify an image is $120 \mathrm{~ms}$ with the best inference time being $115 \mathrm{~ms}$. The quick response time of

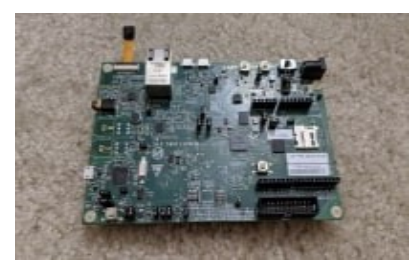

Fig. 4. NXP i.MX RT1060 board 


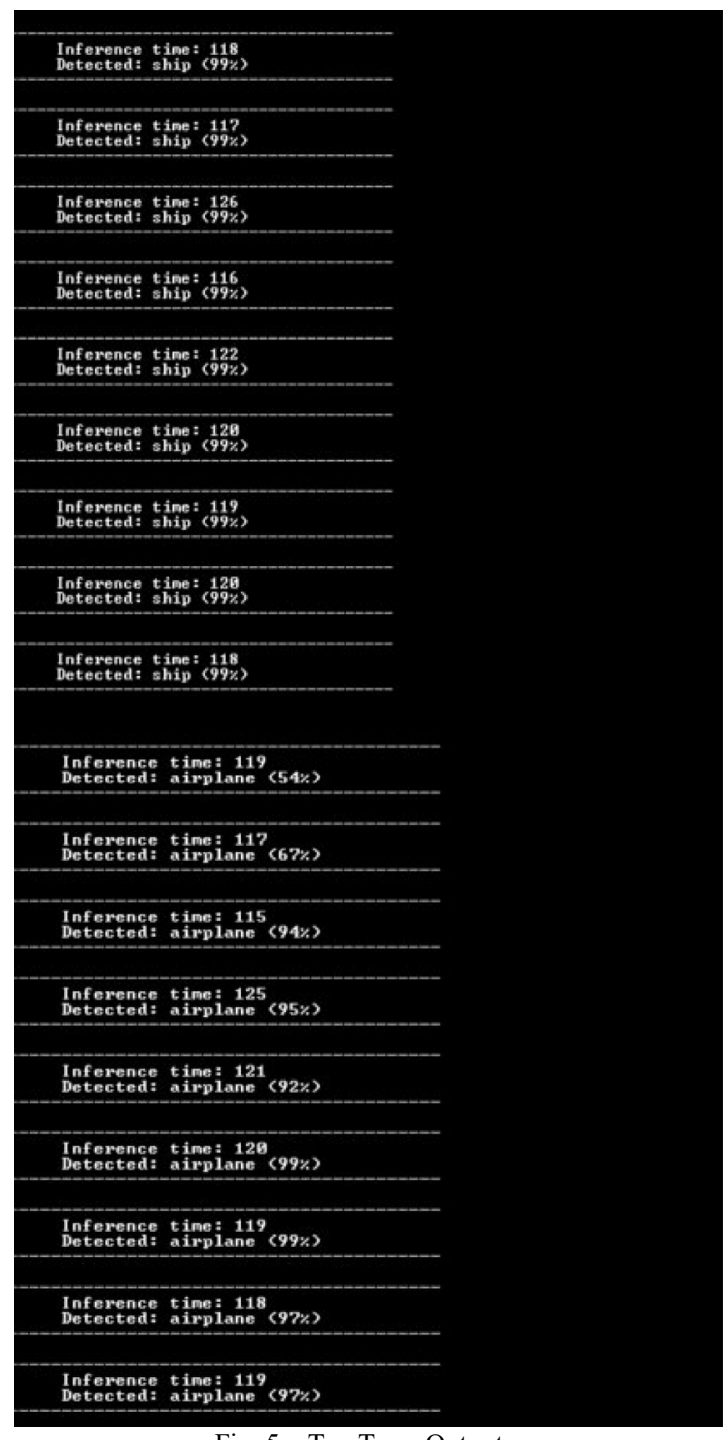

Fig. 5. TeraTerm Output

this model on the above-mentioned processor makes it useful and reliable in real-time computer vision applications for autonomous vehicles. The future scope of this research involves the utilization of the DSE technique again and increasing the accuracy of the model beyond while keeping the size of the model the same. The model size can be reduced further with the help of techniques like Deep compression, Pruning and Architecture tuning, but it may lead to a compromise in the overall accuracy. The Ultra-thin MobileNet model can be deployed to other efficient processors of the NXP i.MX RT family, NXP Bluebox 2.0, etc and developing other interesting computer vision applications like real-time object detection, object tracking, object segmentation, etc with better inference times.

\section{ACKNOWLEDGMENT}

The authors would like thank NXP semiconductors and the IoT collaboratory under Purdue School of Engineering and Technology, IUPUI for supporting this research work.

\section{REFERENCES}

[1] Iandola, F. N., Han, S., Moskewicz, M. W., Ashraf, K., Dally, W. J. and Keutzer, K., (2016). SqueezeNet: AlexNet-level accuracy with 50x fewer parameters and $0.5 \mathrm{MB}$ model size. arXiv preprint arXiv:1602.07360

[2] Amir Gholami, Kiseok Kwon, Bichen Wu, Zizheng Tai, Xiangyu Yue, Peter Jin, Sicheng Zhao, Kurt Keutzer, (2018). SqueezeNext: HardwareAware Neural Network Design. arXiv preprint arXiv: 1803.10615

[3] Andrew G. Howard, Menglong Zhu, Bo Chen, Dmitry Kalenichenko, Weijun Wang, Tobias Weyand, Marco Andreetto, Hartwig Adam (2017). MobileNets: Efficient Convolutional Neural Networks for Mobile Vision Applications. arXiv preprint arXiv:1704.04861

[4] Christian Szegedy, Wei Liu, Yangqing Jia, Pierre Sermanet, Scot Reed, Dragomir Anguelov, Dumitru Erhan, Vincent Vanhoucke, Andrew Rabinovich (2014). Going Deeper with Convolutions. arXiv preprintarXiv: 1409.4842

[5] Mark Sandler, Andrew Howard, Menglong Zhu, Andrey Zhmoginov, Liang-Chieh Chen (2019). MobileNetV2: Inverted Residuals and Linear Bottlenecks. arXiv preprint arXiv: 1801.04381v4

[6] Md Zahangir Alom, Tarek M. Taha, Christopher Yakopcic, Stefan Westberg, Paheding Sidike, Mst Shamima Nasrin, Brian C Van Esesn, Abdul A S. Awwal, Vijayan K. Asari (2018). The History Began from AlexNet: A Comprehensive Survey on Deep Learning

[7] Ji Lin, Yongming Rao, Jiwen Lu, Jie Zhou (2017). Runtime Neura Pruning. 31st Conference on Neural Information Processing Systems, Long Beach, CA, USA.

[8] Xiangyu Zhang, Xinyu Zhou, Mengxiao LinJian, Sun. ShuffleNet: An Extremely Efficient Convolutional Neural Network for Mobile. arXiv preprint arXiv:1707.01083v2, 2017

[9] Francois Chollet (2017). Xception: Deep Learning with DepthwiseSeparable Convolutions. arXiv preprint arXiv: 1610.02357

[10] Ilya Sutskever, James Martens, George Dahl, Georey Hinton (2013) On the importance of initialization and momentum in deep learning.http://proceedings.mlr.press/v28/sutskever13.pdf

[11] Gao Huang, Shichen Liu, Laurens van der Maaten. CondenseNet: An Efficient DenseNet using Learned Group Convolutions. arXiv preprint arXiv: 1711.09224, 2017

[12] Diederik Kingma and Jimmy Ba. Adam: A method for stochastic optimization. arXiv preprint arXiv:1412.6980, 2014

[13] Zhun Zhong, Liang Zheng, Guoliang Kang, Shaozi Li, Yi Yang (2017).Random Erasing Data Augmentation. arXiv preprint arXiv: 1708.04896

[14] Hong-Yen Chen and Chung-Yen Su. An Enhanced Hybrid MobileNet. arXiv preprint arXiv: 1712.04698

[15] Prajit Ramachandran, Barret Zoph, Quoc V. Le (2017).Searching for Activation Functions. arXiv preprint arXiv: 1710.05941

[16] Abien Fred M. Agarap (2019). Deep Learning using Rectified Linear Units (ReLU). arXiv preprint arXiv: $1803.08375 \mathrm{v} 2$

[17] Barret Zoph, Ekin D. Cubuk, Golnaz Ghiasi, Tsung-Yi Lin, Jonathon Shlens, Quoc V (2019). Le. Learning Data Augmentation. arXiv preprint arXiv: 1906.11172

[18] Steffen Eger, Paul Youssef, Iryna Gurevych (2019). Is it Time to Swish? Comparing Deep Learning Activation Functions Across NLP tasks. arXiv preprint arXiv: 1901.02671

[19] Chigozie Enyinna Nwankpa, Winifred Ijomah, Anthony Gachagan, and Stephen Marshall (2018). Activation Functions: Comparison of Trends in Practice and Research for Deep Learning. arXiv preprint arXiv: $1811.03378 \mathrm{v} 1$

[20] Timothy Dozat (2016). INCORPORATING NESTEROV MOMENTUMINTO ADAM. Workshop track - ICLR 2016.

[21] T. Tieleman and G. Hinton. Lecture 6.5-rmsprop: Divide the gradientby a running average of its recent magnitude. COURSERA: Neural Networks for Machine Learning, 4(2), 2012.

[22] Stefan Elfwing, Eiji Uchibe, and Kenji Doya. Sigmoid-Weighted Linear Units for Neural Network Function Approximation in Reinforcement Learning. arXiv preprint arXiv: 1702.03118, 2017

[23] Aleksandar Botev, Guy Lever, David Barber (2016). Nesterovs Accelerated Gradient and Momentum as approximations to Regularised Update Descent. arXiv preprint arXiv: 1607.01981

[24] Timothy Dozat (2016). INCORPORATING NESTEROV MOMENTUM INTO ADAM. Workshop track - ICLR 2016.

[25] Yi Zhou, Junjie Yang, Huishuai Zhang, Yingbin Liang, Vahid Tarokh. SGD CONVERGES TO GLOBAL MINIMUM IN DEEP LEARN- 
INGVIA STAR-CONVEXPATH. arXiv preprint arXiv :1901.00451, 2019

[26] Song Han, Huizi Mao, William J. Dally (2015). Deep Compression: Compressing Deep Neural Network with Pruning, Trained Quantization and Huffman Coding- ICLR 2015

[27] Ilya Sutskever, James Martens, George Dahl, Geoffrey Hinton. On the importance of initialization and momentum in deep learning. ICML'13 Proceedings of the 30th International Conference on International Conference on Machine Learning.

[28] Ido Freeman, Lutz Roese-Koerne, Anton Kummert. EffNet: AN EFFICIENT STRUCTURE FOR CONVOLUTIONAL NEURAL NETWORKS. arXiv preprint arXiv: 1801.06434, 2018

[29] Xiangyu Zhang, Xinyu Zhou, Mengxiao LinJian, Sun. ShuffleNet: An Extremely Efficient Convolutional Neural Network for Mobile. arXiv preprint arXiv:1707.01083v2, 2017 